\title{
Bottom-Up, On-Surface-Synthesized Armchair Graphene Nanoribbons for Ultra-High-Power Micro-Supercapacitors
}

\author{
Zhaoyang Liu, ${ }^{\perp}$ Zongping Chen, ${ }^{\perp}$ Can Wang, ${ }^{\perp}$ Hai I. Wang, Michael Wuttke, Xiao-Ye Wang, \\ Mischa Bonn, Lifeng Chi, Akimitsu Narita,* and Klaus Müllen*
}

Cite This: J. Am. Chem. Soc. 2020, 142, 17881-17886

Read Online

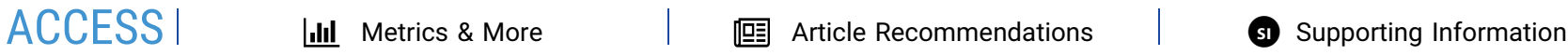

ABSTRACT: Bottom-up-synthesized graphene nanoribbons (GNRs) with excellent electronic properties are promising materials for energy storage systems. Herein, we report bottom-up-synthesized GNR films employed as electrode materials for microsupercapacitors (MSCs). The micro-device delivers an excellent volumetric capacitance and an ultra-high power density. The electrochemical performance of MSCs could be correlated with the charge carrier mobility within the differently employed GNRs, as determined by pump-probe terahertz spectroscopy studies.

$\mathrm{T}$ he past decades have witnessed an urgent need for developing reliable, efficient, and eco-friendly approaches for energy storage systems (ESSs). ${ }^{1,2}$ Following the current trend of portable and wearable electronics, the demand for ultrathin, flexible, and powerful on-chip micro-scale ESSs has been growing rapidly. ${ }^{3-5}$ Among them, micro-supercapacitors (MSCs) with high charging/discharging rates, long cycle lives, high areal/volumetric capacitance, and high power densities have great potential to complement or even replace microbatteries. ${ }^{6,7}$ The recently introduced graphene-based MSCs with planar interdigitated micro-electrodes combine the best of graphene's advantages, i.e., its large specific surface area and high intrinsic capacitance as well as excellent mechanical and electronic properties. ${ }^{8-13}$ Moreover, the open-edge-normalized capacitance of graphene-based electrode materials is higher than that of basal planes. ${ }^{14-17}$ In this regard, narrow graphene nanoribbons (GNRs), defined as nanometer-wide strips of graphene, ${ }^{18,19}$ are promising for MSC applications.

Recently, several top-down approaches have been demonstrated to fabricate GNRs, e.g., unzipping of carbon nanotubes, ${ }^{20}$ photolithography patterning of graphene or graphene oxide, ${ }^{17,21,22}$ or activation of carbon nanorods from metalorganic frameworks. ${ }^{23}$ However, it remains a formidable challenge to fabricate GNRs with well-defined width and edge structures via such top-down approaches. ${ }^{24}$ In contrast, a bottom-up chemical approach allows for the synthesis of GNRs with precisely controlled width and edge structures in solution or on a metal surface. ${ }^{25-35}$ Solution-synthesized GNRs tend to aggregate and are difficult to process on different substrates, $^{18,36}$ but the on-surface synthesis allows for the fabrication and transfer of GNR films onto an arbitrary substrate without the aggregation problem. ${ }^{34,35,37}$ While most of the previous on-surface syntheses of GNRs have been carried out under ultra-high-vacuum conditions, which limit the scalability of the synthesis, recent studies have demonstrated high-throughput, on-surface synthesis of GNRs using chemical vapor deposition (CVD). ${ }^{24,38-41}$ The CVD-grown $N$ $=9$ armchair GNRs showed high photoconductivity with estimated intrinsic mobilities of $\sim 350 \mathrm{~cm}^{2} \mathrm{~V}^{-1} \mathrm{~s}^{-1}$. ${ }^{39}$ This result also marks the potential of GNRs as novel materials for electrochemical storage applications, where sufficiently high charge mobility is required. ${ }^{2,38,39}$ Nevertheless, the use of bottom-up-synthesized GNRs in ESSs has rarely been explored.

Herein, we report the unprecedented use of bottom-upsynthesized GNR films as MSC electrode materials. Armchair graphene nanoribbons (AGNRs) with three different widths ( $n$-AGNRs, with $n=5,7$, and 9, indicating the width by the number of carbon atoms, see Figure $1 \mathrm{~b}$ ) were prepared by the CVD method and examined. Among the three AGNRs, we reveal that electrodes based on the narrowest 5-AGNR deliver

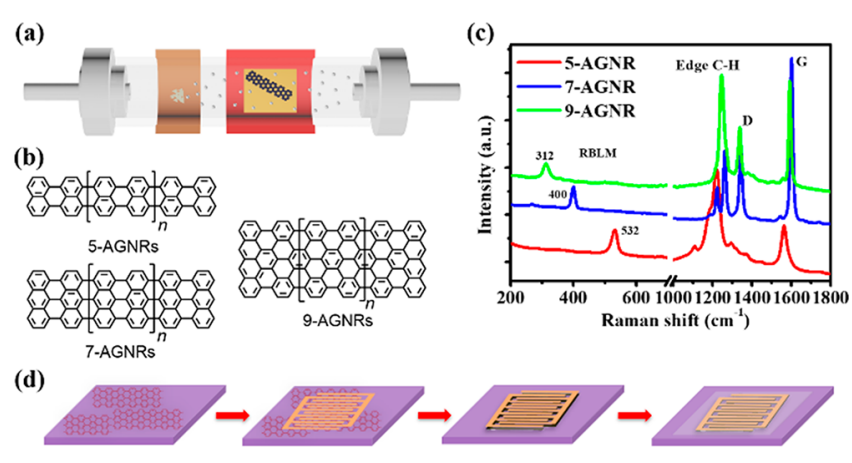

Figure 1. (a) Schematic illustration of the growth of AGNRs via CVD. (b) Molecular structures and (c) Raman spectra of 5-AGNR, 7AGNR, and 9-AGNR. (d) Schematic illustration of the device fabrication of MSCs based on GNR films.

Received: June 5, 2020

Published: October 6, 2020 
(a)

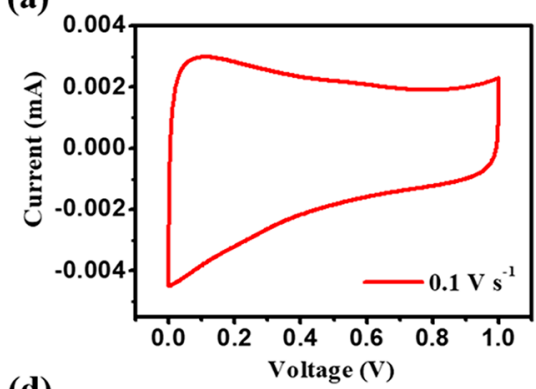

(d)

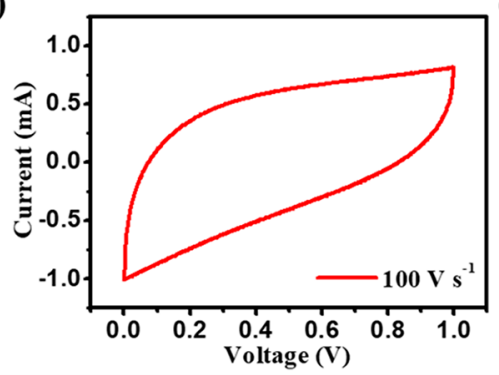

(b)

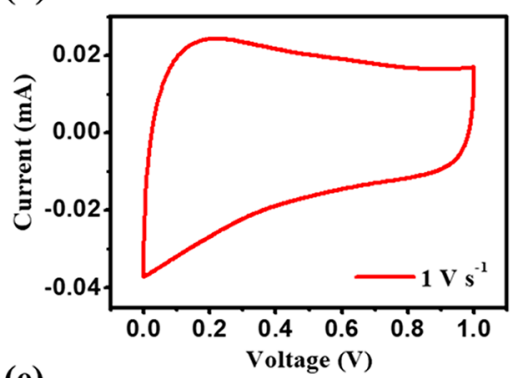

(e)

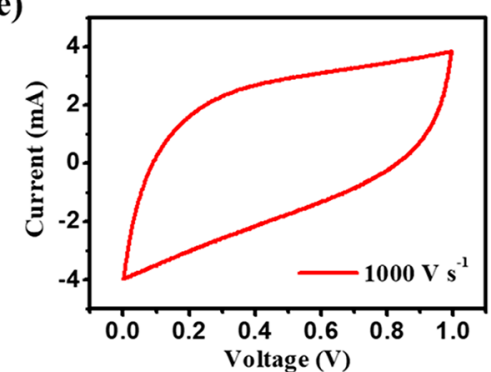

(c)
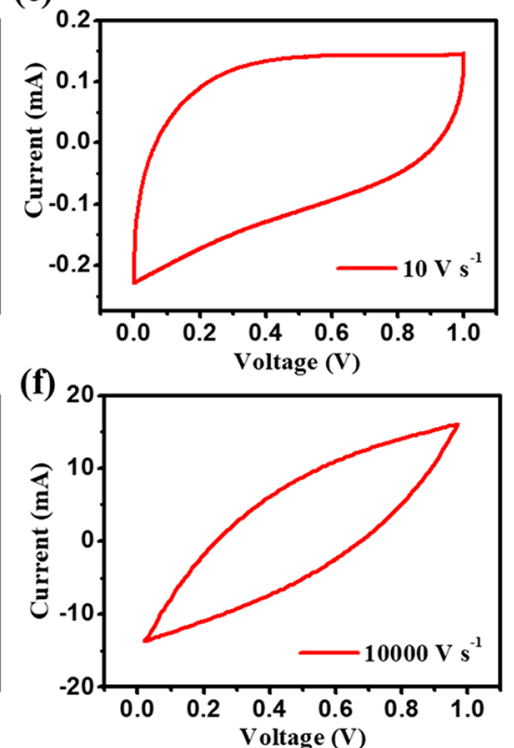

Figure 2. CV curves of a micro-supercapacitor based on 5 layers of 5-AGNR films, performed at different scan rates ranging from 0.1 to $10000 \mathrm{~V}$ $\mathrm{s}^{-1}$, for a potential window from 0 to $1 \mathrm{~V}$.

the highest MSC performance, with an excellent volumetric capacitance of $307 \mathrm{~F} \mathrm{~cm}^{-3}$ and an ultra-high power density of $2000 \mathrm{~W} \mathrm{~cm}{ }^{-3}$, outperforming most state-of-the-art MSCs based on graphene and other related nanomaterials. Furthermore, ultrafast terahertz $(\mathrm{THz})$ spectroscopy analyses demonstrated the highest photoconductivity for 5-AGNR compared to those of 7- and 9-AGNRs, in excellent correlation with the MSC results. Such promising electrochemical performance suggests the high potential of GNRs as electrode materials for ESSs.

9-AGNR, 7-AGNR, and 5-AGNR films were prepared using an ambient-pressure CVD setup, following our previous reports (Figure 1a,b). ${ }^{38,39}$ These AGNRs belong to three different subfamilies of AGNRs with $N=3 n, 3 n+1$, and $3 n+2$, respectively, having distinct bandgaps in the order of 5-AGNR $<$ 9-AGNR < 7-AGNR. ${ }^{42}$ The formation of the AGNRs was verified by Raman spectroscopy, exhibiting the typical G, D, and $\mathrm{C}-\mathrm{H}$ peaks (Figure 1c). ${ }^{2,38,39,43}$ Importantly, different AGNRs were identified on the basis of the characteristic radial breathing-like mode (RBLM), i.e., $532 \mathrm{~cm}^{-1}$ for 5-AGNR, 400 $\mathrm{cm}^{-1}$ for 7 -AGNR, and $312 \mathrm{~cm}^{-1}$ for 9-AGNR. ${ }^{19,38,39,44,45}$ AGNR films thus grown on the $\mathrm{Au} /$ mica substrates were then transferred onto $\mathrm{SiO}_{2} / \mathrm{Si}$ substrates through a lift-off process with the help of poly(methyl methacrylate) (PMMA) supporting layer. ${ }^{46,47}$ Analyses of a transferred 5-AGNR film by transmission electron microscopy (Figure S1), atomic force microscopy, and scanning electron microscopy (Figure S2) indicated homogeneous films with $\sim 1 \mathrm{~nm}$ thickness. For the fabrication of all-solid-state MSCs with an in-plane device geometry, 5 layers of CVD-grown AGNR films were sequentially transferred onto $\mathrm{SiO}_{2} / \mathrm{Si}$ substrates (Figure 1d), followed by gold deposition through shadow masks acting as current collectors (Figure S3). $\mathrm{A} \mathrm{H}_{2} \mathrm{SO}_{4} /$ poly(vinyl alcohol) $\left(\mathrm{H}_{2} \mathrm{SO}_{4} / \mathrm{PVA}\right)$ gel electrolyte was employed.

Thus-fabricated AGNRs-based MSCs were analyzed by cyclic voltammetry $(\mathrm{CV})$ at different scan rates to evaluate the overall electrochemical performance (Figures 2, S4, and S5). The obtained CV curves suggest that these devices can be operated from 0.1 to $10000 \mathrm{~V} \mathrm{~s}^{-1}$. Notably, the maximum operating speed for such AGNRs-based MSCs was revealed to be 4 orders of magnitude higher than that of conventional supercapacitors based on sandwiched structures, and even 1 order of magnitude higher than that of state-of-the-art, highpower MSCs based on graphene or other carbon nanomaterials (Table S1). These results mark the ultrafast charging/ discharging capabilities of the AGNRs-based MSCs. ${ }^{10}$ Between the voltage window of 0 and $1 \mathrm{~V}$, the $\mathrm{CV}$ curves are all nearly rectangularly shaped, even at a very high scan rate of $1000 \mathrm{~V}$ $\mathrm{s}^{-1}$. This indicates a pronounced electrical double-layer capacitance from the AGNRs with preserved electronic properties, which is inherent to their graphene structure. One of the major differences between the CV curves based on different AGNRs is the charge/discharge current values, where the 5-AGNR-based MSC is superior to those based on the other two AGNRs. Especially, at a scan rate of $0.1 \mathrm{~V} \mathrm{~s}^{-1}$, a maximum volumetric capacitance of $307 \mathrm{~F} \mathrm{~cm}^{-3}$ is achieved based on a $\sim 5 \mathrm{~nm}$ thick 5-AGNR film electrode (results for a MSC with a different film thickness are shown in Figure S6), whereas $180 \mathrm{~F} \mathrm{~cm}^{-3}$ is obtained based on 7-AGNR and $200 \mathrm{~F}$ $\mathrm{cm}^{-3}$ based on 9-AGNR with the same film thickness.

Figure $3 \mathrm{a}$ plots the evolution of the volumetric capacitance of the three AGNR electrodes-based MSCs at different scan rates. The general electrochemical performance regarding volumetric capacitance falls in the following sequence, 5AGNR > 9-AGNR > 7-AGNR, which exceeding the performance of most state-of-the-art graphene and other carbon materials (Table S1) and comparable to the highest values reported to date, such as $359 \mathrm{~F} \mathrm{~cm}^{-3}$ based on electrophoretically deposited $\mathrm{rGO},{ }^{13} 180$ and $410 \mathrm{~F} \mathrm{~cm}^{-3}$ based on carbidederived carbon, ${ }^{48,49}$ and $582 \mathrm{~F} \mathrm{~cm}^{-3}$ based on sulfur-doped graphene. ${ }^{9}$ The excellent volumetric capacitance might be attributed to the higher density of edge structures of AGNRs, which allows faster ion transport and more storage charges. ${ }^{50}$ These results illustrate the great potential of GNRs as material for efficient on-chip EESs. Furthermore, the MSCs all show $\sim 10 \%$ capacitance retention over a wide operating rate of $0.1-$ $1000 \mathrm{~V} \mathrm{~s}^{-1}$. Such excellent rate capabilities are essential for powerful MSCs. Figure 3b suggests a nearly linear correlation 


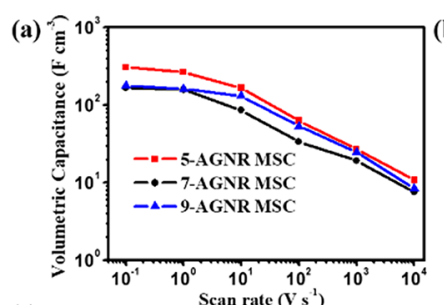

(c)
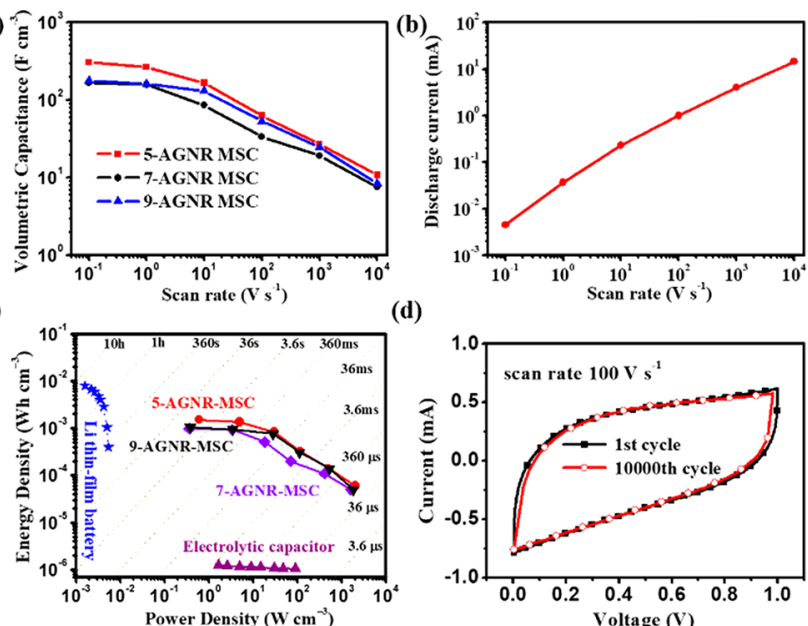

Figure 3. (a) Evolution of the volumetric capacitance versus scan rate based on 5 layers of 5-AGNR, 7-AGNR, and 9-AGNR films. (b) Discharge current of 5-AGNR-based MSC device as a function of scan rate. (c) Ragone plot of commercially available Li thin-film battery and electrolytic capacitor compared with different AGNR-based MSCs. (d) CV curves of the 1st and 10000 th cycles of a 5-AGNRbased MSC device, at a high operating speed of $100 \mathrm{~V} \mathrm{~s}^{-1}$, showing capacitance retention over $95 \%$.

between the logarithmic discharge current and scan rate from 0.1 to $10000 \mathrm{~V} \mathrm{~s}^{-1}$, indicating the ultra-high-power delivery of 5-AGNR-based MSCs. Electrochemical impedance spectroscopy (EIS) of 5-AGNR MSCs is measured in a frequency range from $0.01 \mathrm{~Hz}$ to $100 \mathrm{kHz}$ (Figure S7). The characteristics of low ESR and ultrasmall charge transfer resistance, as well as extremely short time constant, validate the excellent charge and discharge capability of the 5-AGNR MSCs.

A maximum power density of $2000 \mathrm{~W} \mathrm{~cm}^{-3}$ is achieved for the 5-AGNR-based MSC, larger than the values obtained for the other two AGNRs, namely $1600 \mathrm{~W} \mathrm{~cm}^{-3}$ for 7-AGNR and $1800 \mathrm{~W} \mathrm{~cm}^{-3}$ for 9-AGNR. Figure $3 \mathrm{c}$ shows the Ragone plot of the energy and power densities of MSCs based on the employed AGNRs. Compared with those of a commercially available lithium thin-film battery $(4 \mathrm{~V} / 500 \mu \mathrm{Ah})$ and an aluminum electrolytic capacitor $(3 \mathrm{~V} / 300 \mu \mathrm{F})$, the power density of AGNRs based MSCs is 2 orders of magnitude higher than that of conventional supercapacitors with sandwiched structures, and over 1 order of magnitude higher than that of aluminum electrolytic capacitors. Such comparisons with reallife ESSs clearly illustrate the huge potential of AGNRs-based MSCs, especially 5-AGNR. Meanwhile, the cycling stability study of 5-AGNR MSCs examined at a high operating speed of $100 \mathrm{~V} \mathrm{~s}^{-1}$ reveals capacitance retention over $95 \%$ after 10000 cycles (Figure 3d), which further demonstrates the applicability of 5-AGNR MSCs.

To elucidate the role of electronic properties of GNRs on the MSC device performance, we employed $\mathrm{THz}$ spectroscopy to characterize the conductivity of photogenerated carriers in AGNRs. ${ }^{38,39}$ To this end, AGNR films were transferred on $\mathrm{SiO}_{2}$ substrates, and charge carriers were optically generated in AGNRs by a laser pulse with $\sim 50$ fs duration and photon energy of $3.1 \mathrm{eV}$. This photon energy well exceeds the optical bandgaps of AGNRs used here (0.8, 1.0, and $1.6 \mathrm{eV}$ for 5-, 9-, and 7-AGNRs, respectively) so that initially charge carriers appear as free carriers following photoexcitation. In the Supporting Information, we provide a detailed discussion on the working principle of the $\mathrm{THz}$ spectroscopy. In Figure 4, we show the time-dependent $\mathrm{THz}$ conductivity $\sigma$ of 5-AGNRs and

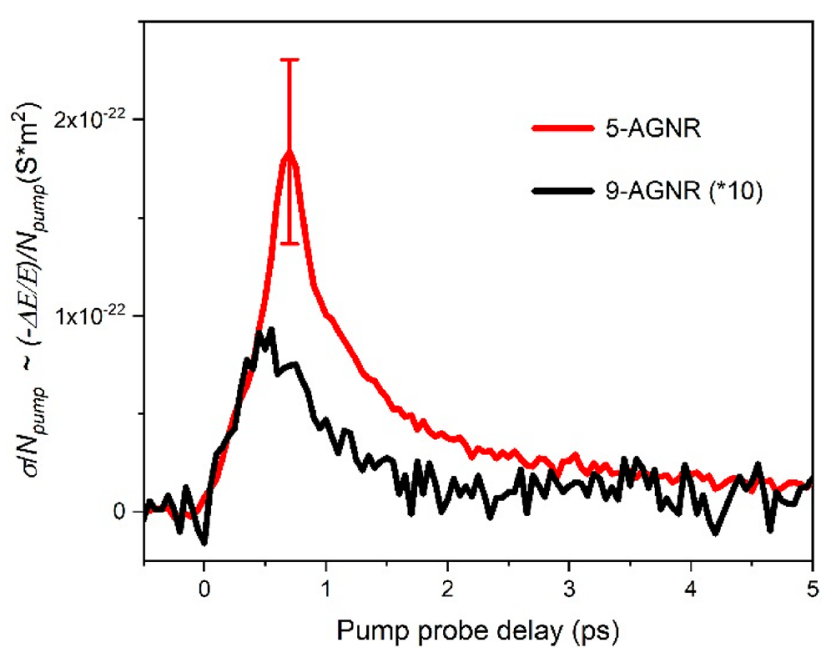

Figure 4. Photoconductivity $\sigma$ due to $400 \mathrm{~nm}$ photoinjection of charge carriers, divided by the absorbed photon flux $N_{\text {pump. }}$. The photoconductivity $\sigma$ is proportional to the relative transmission change of the $\mathrm{THz}$ field $(-\Delta E / E)$ following photoexcitation. The conductivity of the 9-AGNR is scaled up by a factor of 10 for a better comparison to that of 5-AGNR. The root-mean-square variation of the peak conductivity was obtained from measurements at 6 different sample spots (see Figure S8a).

9-AGNRs normalized to the absorbed pump photon density. Following the quasi-instantaneous optical charge carrier generation, we observe a rapid decay in $\sim 2 \mathrm{ps}$, which has been assigned to the formation of excitons from the initially free carriers. ${ }^{51}$ Importantly, the relative magnitudes of the signals testify to a remarkably ( $\sim 20$-fold) higher charge carrier mobility of 5-AGNRs compared to that of 9-AGNRs. Previous work employing the same approach has shown that 9-AGNRs is about $20 \%$ more conductive than 7 -AGNRs. ${ }^{39}$ This leads to the following trend of charge mobility $\mu$ in AGNRs: 5-AGNRs $\gg$ 9-AGNRs $>7$-AGNRs $\left(\mu=e \tau / m^{*}\right.$, with $e$ the electron charge, $\tau$ the charge scattering time, and $m^{*}$ the effective mass of the charge carriers). The charge scattering time for various GNRs has previously been determined to be in the range of 20-30 fs. ${ }^{52}$ The main difference in mobility seems, therefore, to originate from the effective mass difference of AGNRs, perfectly in line with the effective masses obtained from previous calculations $\left(0.01,0.091\right.$, and $0.21 m_{0}$ for 5-, 9-, and 7-AGNR, respectively). ${ }^{53}$ In line with our discussion here, a recent electrical transport work based on AGNR FET devices has demonstrated that the charge carrier mobility in 5-AGNRs is 1 order of magnitude higher than that of 9-AGNRs. ${ }^{54}$ Thus, we believe that defects do not play a major role for transport. Interestingly, the trend in charge carrier mobility in the GNR electrodes reflects the trend observed in the MSC electrochemical performances. The correlation between the charge carrier effective mass, the photoconductivity, and the volumetric capacitance illustrates the critical role of the intrinsic electronic properties of GNRs on their performance in electrochemical energy storage applications. Such correlation is perhaps not surprising: The GNRs used here are grown by CVD with a limited length up to $\sim 40 \mathrm{~nm}$. As such, electrical transport through the GNR network may become the limiting step for charge injections. In that case, the high conductivity in 
5-AGNRs will facilitate the electrical transport process and lead to a marked improvement in the electrochemical performance.

In conclusion, we have successfully used bottom-up, onsurface-synthesized AGNRs for high-performance MSCs. The AGNRs-based MSCs demonstrate promising overall performance, such as ultra-high operating speeds, volumetric capacitances, and power densities, outperforming state-of-theart MSCs based on other graphene materials. The AGNRsbased MSC devices' performance correlates well with the charge carrier mobility indicated by $\mathrm{THz}$ studies, i.e., 5AGNRs $\gg$ 9-AGNRs $>$ 7-AGNRs. Future optimization of the CVD process for ultra-long AGNRs growth and a better design concept for metallic AGNRs, as well as a scale-up of defect-free AGNRs production via solution-phase synthesis accompanied by suitable processing approaches, may pave the way for such novel one-dimensional carbon materials being employed as practical ESSs, even in flexible and wearable forms.

\section{ASSOCIATED CONTENT}

\section{SI Supporting Information}

The Supporting Information is available free of charge at https://pubs.acs.org/doi/10.1021/jacs.0c06109.

Materials, methods, device geometry, and CV information (PDF)

\section{AUTHOR INFORMATION}

\section{Corresponding Authors}

Akimitsu Narita - Max Planck Institute for Polymer Research, 55128 Mainz, Germany; 이이.org/0000-0002-3625522X; Email: narita@mpip-mainz.mpg.de

Klaus Müllen - Max Planck Institute for Polymer Research, 55128 Mainz, Germany; Institute of Physical Chemistry, Johannes Gutenberg-Universität Mainz, 55128 Mainz, Germany; ○ orcid.org/0000-0001-6630-8786; Email: muellen@mpip-mainz.mpg.de

\section{Authors}

Zhaoyang Liu - Max Planck Institute for Polymer Research, 55128 Mainz, Germany; 이이.org/0000-0001-7972-3452

Zongping Chen - Max Planck Institute for Polymer Research, 55128 Mainz, Germany

Can Wang - Jiangsu Key Laboratory for Carbon Based Functional Materials \& Devices, Institute of Functional Nano \& Soft Materials (FUNSOM), Soochow University, Suzhou 215123, P. R. China

Hai I. Wang - Max Planck Institute for Polymer Research, 55128 Mainz, Germany; 이이.org/0000-0003-0940-3984

Michael Wuttke - Max Planck Institute for Polymer Research, 55128 Mainz, Germany

Xiao-Ye Wang - Max Planck Institute for Polymer Research, 55128 Mainz, Germany; 이이이.org/0000-0003-3540-0277

Mischa Bonn - Max Planck Institute for Polymer Research, 55128 Mainz, Germany; 이이이.org/0000-0001-6851-8453

Lifeng Chi - Jiangsu Key Laboratory for Carbon Based Functional Materials \& Devices, Institute of Functional Nano \& Soft Materials (FUNSOM), Soochow University, Suzhou 215123, P. R. China; (1) orcid.org/0000-0003-3835-2776

Complete contact information is available at:

https://pubs.acs.org/10.1021/jacs.0c06109

\section{Author Contributions}

${ }^{\perp}$ Z.L., Z.C., and C.W. contributed equally to this work.

\section{Notes}

The authors declare no competing financial interest.

\section{ACKNOWLEDGMENTS}

We dedicate this paper to the memory of our colleague Paniz Soltani. We acknowledge financial support from the Max Planck Society, EU Projects through the FET-Proactive Project "MoQuaS" (N.610449), Graphene Flagship (No. CNECTICT-604391), and Horizon 2020 research and innovation programme GrapheneCore1 (696656) and GrapheneCore2 (785219).

\section{REFERENCES}

(1) Bonaccorso, F.; Colombo, L.; Yu, G.; Stoller, M.; Tozzini, V.; Ferrari, A. C.; Ruoff, R. S.; Pellegrini, V. Graphene, related twodimensional crystals, and hybrid systems for energy conversion and storage. Science 2015, 347 (6217), 1246501.

(2) Wang, G.; Zhang, L.; Zhang, J. A review of electrode materials for electrochemical supercapacitors. Chem. Soc. Rev. 2012, 41 (2), 797-828.

(3) Shao, Y.; El-Kady, M. F.; Wang, L. J.; Zhang, Q.; Li, Y.; Wang, H.; Mousavi, M. F.; Kaner, R. B. Graphene-based materials for flexible supercapacitors. Chem. Soc. Rev. 2015, 44, 3639-3665.

(4) Beidaghi, M.; Gogotsi, Y. Capacitive energy storage in microscale devices: Recent advances in design and fabrication of microsupercapacitors. Energy Environ. Sci. 2014, 7 (3), 867-884.

(5) Zuo, Z.; Li, Y. Emerging Electrochemical Energy Applications of Graphdiyne. Joule 2019, 3 (4), 899-903.

(6) Wu, Z.-S.; Feng, X.; Cheng, H.-M. Recent advances in graphenebased planar micro-supercapacitors for on-chip energy storage. Natl. Sci. Rev. 2014, 1 (2), 277-292.

(7) Kyeremateng, N. A.; Brousse, T.; Pech, D. Microsupercapacitors as miniaturized energy-storage components for on-chip electronics. Nat. Nanotechnol. 2017, 12 (1), 7-15.

(8) Wu, Z. S.; Zheng, Y.; Zheng, S.; Wang, S.; Sun, C.; Parvez, K.; Ikeda, T.; Bao, X.; Müllen, K.; Feng, X. Stacked-Layer Heterostructure Films of 2D Thiophene Nanosheets and Graphene for HighRate All-Solid-State Pseudocapacitors with Enhanced Volumetric Capacitance. Adv. Mater. 2017, 29, 1602960.

(9) Wu, Z.-S.; Tan, Y.-Z.; Zheng, S.; Wang, S.; Parvez, K.; Qin, J.; Shi, X.; Sun, C.; Bao, X.; Feng, X.; Müllen, K. Bottom-Up Fabrication of Sulfur-Doped Graphene Films Derived from Sulfur-Annulated Nanographene for Ultrahigh Volumetric Capacitance Micro-Supercapacitors. J. Am. Chem. Soc. 2017, 139, 4506-4512.

(10) Wu, Z. S.; Parvez, K.; Feng, X.; Müllen, K. Graphene-based inplane micro-supercapacitors with high power and energy densities. Nat. Commun. 2013, 4, 2487.

(11) El-Kady, M. F.; Kaner, R. B. Scalable fabrication of high-power graphene micro-supercapacitors for flexible and on-chip energy storage. Nat. Commun. 2013, 4, 1475.

(12) Gao, W.; Singh, N.; Song, L.; Liu, Z.; Reddy, A. L. M.; Ci, L.; Vajtai, R.; Zhang, Q.; Wei, B.; Ajayan, P. M. Direct laser writing of micro-supercapacitors on hydrated graphite oxide films. Nat. Nanotechnol. 2011, 6 (8), 496-500.

(13) Niu, Z.; Zhang, L.; Liu, L.; Zhu, B.; Dong, H.; Chen, X. AllSolid-State Flexible Ultrathin Micro-Supercapacitors Based on Graphene. Adv. Mater. 2013, 25 (29), 4035-4042.

(14) Yoo, J. J.; Balakrishnan, K.; Huang, J.; Meunier, V.; Sumpter, B. G.; Srivastava, A.; Conway, M.; Mohana Reddy, A. L.; Yu, J.; Vajtai, R.; Ajayan, P. M. Ultrathin Planar Graphene Supercapacitors. Nano Lett. 2011, 11 (4), 1423-1427.

(15) Yuan, W.; Zhou, Y.; Li, Y.; Li, C.; Peng, H.; Zhang, J.; Liu, Z.; Dai, L.; Shi, G. The edge- and basal-plane-specific electrochemistry of a single-layer graphene sheet. Sci. Rep. 2013, 3, 2248.

(16) Xiang, Z.; Dai, Q.; Chen, J.-F.; Dai, L. Edge Functionalization of Graphene and Two-Dimensional Covalent Organic Polymers for Energy Conversion and Storage. Adv. Mater. 2016, 28 (29), 62536261. 
(17) Qi, D.; Liu, Z.; Liu, Y.; Leow, W. R.; Zhu, B.; Yang, H.; Yu, J.; Wang, W.; Wang, H.; Yin, S.; Chen, X. Suspended Wavy Graphene Microribbons for Highly Stretchable Microsupercapacitors. Adv. Mater. 2015, 27, 5559-5566.

(18) Narita, A.; Feng, X.; Hernandez, Y.; Jensen, S. A.; Bonn, M.; Yang, H.; Verzhbitskiy, I. A.; Casiraghi, C.; Hansen, M. R.; Koch, A. H. R.; Fytas, G.; Ivasenko, O.; Li, B.; Mali, K. S.; Balandina, T.; Mahesh, S.; De Feyter, S.; Müllen, K. Synthesis of structurally welldefined and liquid-phase-processable graphene nanoribbons. Nat. Chem. 2014, 6 (2), 126-132.

(19) Cai, J.; Ruffieux, P.; Jaafar, R.; Bieri, M.; Braun, T.; Blankenburg, S.; Muoth, M.; Seitsonen, A. P.; Saleh, M.; Feng, X.; Mullen, K.; Fasel, R. Atomically precise bottom-up fabrication of graphene nanoribbons. Nature 2010, 466 (7305), 470-473.

(20) Zhang, C.; Peng, Z.; Lin, J.; Zhu, Y.; Ruan, G.; Hwang, C.-C.; Lu, W.; Hauge, R. H.; Tour, J. M. Splitting of a Vertical Multiwalled Carbon Nanotube Carpet to a Graphene Nanoribbon Carpet and Its Use in Supercapacitors. ACS Nano 2013, 7 (6), 5151-5159.

(21) Han, M. Y.; Özyilmaz, B.; Zhang, Y.; Kim, P. Energy Band-Gap Engineering of Graphene Nanoribbons. Phys. Rev. Lett. 2007, 98 (20), 206805.

(22) Chen, Z.; Lin, Y.-M.; Rooks, M. J.; Avouris, P. Graphene nanoribbon electronics. Phys. E 2007, 40 (2), 228-232.

(23) Pachfule, P.; Shinde, D.; Majumder, M.; Xu, Q. Fabrication of carbon nanorods and graphene nanoribbons from a metal-organic framework. Nat. Chem. 2016, 8, 718-724.

(24) Chen, Z.; Zhang, W.; Palma, C.-A.; Lodi Rizzini, A.; Liu, B.; Abbas, A.; Richter, N.; Martini, L.; Wang, X.-Y.; Cavani, N.; Lu, H.; Mishra, N.; Coletti, C.; Berger, R.; Klappenberger, F.; Kläui, M.; Candini, A.; Affronte, M.; Zhou, C.; De Renzi, V.; del Pennino, U.; Barth, J. V.; Räder, H. J.; Narita, A.; Feng, X.; Müllen, K. Synthesis of Graphene Nanoribbons by Ambient-Pressure Chemical Vapor Deposition and Device Integration. J. Am. Chem. Soc. 2016, 138 (47), 15488-15496.

(25) Talirz, L.; Ruffieux, P.; Fasel, R. On-Surface Synthesis of Atomically Precise Graphene Nanoribbons. Adv. Mater. 2016, 28 (29), 6222-6231.

(26) Narita, A.; Feng, X.; Müllen, K. Bottom-Up Synthesis of Chemically Precise Graphene Nanoribbons. Chem. Rec. 2015, 15 (1), 295-309.

(27) Narita, A.; Wang, X.-Y.; Feng, X.; Mullen, K. New advances in nanographene chemistry. Chem. Soc. Rev. 2015, 44 (18), 6616-6643.

(28) Wang, X.-Y.; Narita, A.; Müllen, K. Precision synthesis versus bulk-scale fabrication of graphenes. Nat. Rev. Chem. 2018, 2 (1), 0100.

(29) Zhou, X.; Yu, G. Modified Engineering of Graphene Nanoribbons Prepared via On-Surface Synthesis. Adv. Mater. 2020, 32 (6), 1905957.

(30) Clair, S.; de Oteyza, D. G. Controlling a Chemical Coupling Reaction on a Surface: Tools and Strategies for On-Surface Synthesis. Chem. Rev. 2019, 119 (7), 4717-4776.

(31) Yano, Y.; Mitoma, N.; Ito, H.; Itami, K. A Quest for Structurally Uniform Graphene Nanoribbons: Synthesis, Properties, and Applications. J. Org. Chem. 2020, 85 (1), 4-33.

(32) Yang, W.; Lucotti, A.; Tommasini, M.; Chalifoux, W. A. Bottom-Up Synthesis of Soluble and Narrow Graphene Nanoribbons Using Alkyne Benzannulations. J. Am. Chem. Soc. 2016, 138 (29), 9137-9144.

(33) Mehdi Pour, M.; Lashkov, A.; Radocea, A.; Liu, X.; Sun, T.; Lipatov, A.; Korlacki, R. A.; Shekhirev, M.; Aluru, N. R.; Lyding, J. W.; Sysoev, V.; Sinitskii, A. Laterally extended atomically precise graphene nanoribbons with improved electrical conductivity for efficient gas sensing. Nat. Commun. 2017, 8 (1), 820.

(34) Sun, Q.; Zhang, R.; Qiu, J.; Liu, R.; Xu, W. On-Surface Synthesis of Carbon Nanostructures. Adv. Mater. 2018, 30 (17), 1705630.

(35) Rizzo, D. J.; Veber, G.; Cao, T.; Bronner, C.; Chen, T.; Zhao, F.; Rodriguez, H.; Louie, S. G.; Crommie, M. F.; Fischer, F. R. Topological band engineering of graphene nanoribbons. Nature 2018, 560 (7717), 204-208.
(36) Yano, Y.; Mitoma, N.; Matsushima, K.; Wang, F.; Matsui, K.; Takakura, A.; Miyauchi, Y.; Ito, H.; Itami, K. Living annulative $\pi$ extension polymerization for graphene nanoribbon synthesis. Nature 2019, 571 (7765), 387-392.

(37) Narita, A.; Chen, Z.; Chen, Q.; Müllen, K. Solution and onsurface synthesis of structurally defined graphene nanoribbons as a new family of semiconductors. Chem. Sci. 2019, 10 (4), 964-975.

(38) Chen, Z.; Wang, H. I.; Bilbao, N.; Teyssandier, J.; Prechtl, T.; Cavani, N.; Tries, A.; Biagi, R.; De Renzi, V.; Feng, X.; Kläui, M.; De Feyter, S.; Bonn, M.; Narita, A.; Müllen, K. Lateral Fusion of Chemical Vapor Deposited N = 5 Armchair Graphene Nanoribbons. J. Am. Chem. Soc. 2017, 139 (28), 9483-9486.

(39) Chen, Z.; Wang, H. I.; Teyssandier, J.; Mali, K. S.; Dumslaff, T.; Ivanov, I.; Zhang, W.; Ruffieux, P.; Fasel, R.; Räder, H. J.; Turchinovich, D.; De Feyter, S.; Feng, X.; Kläui, M.; Narita, A.; Bonn, M.; Müllen, K. Chemical Vapor Deposition Synthesis and Terahertz Photoconductivity of Low-Band-Gap N = 9 Armchair Graphene Nanoribbons. J. Am. Chem. Soc. 2017, 139 (10), 36353638.

(40) Sakaguchi, H.; Kawagoe, Y.; Hirano, Y.; Iruka, T.; Yano, M.; Nakae, T. Width-Controlled Sub-Nanometer Graphene Nanoribbon Films Synthesized by Radical-Polymerized Chemical Vapor Deposition. Adv. Mater. 2014, 26 (24), 4134-4138.

(41) Sakaguchi, H.; Song, S.; Kojima, T.; Nakae, T. Homochiral polymerization-driven selective growth of graphene nanoribbons. Nat. Chem. 2017, 9 (1), 57-63.

(42) Magda, G. Z.; Jin, X.; Hagymasi, I.; Vancso, P.; Osvath, Z.; Nemes-Incze, P.; Hwang, C.; Biro, L. P.; Tapaszto, L. Roomtemperature magnetic order on zigzag edges of narrow graphene nanoribbons. Nature 2014, 514 (7524), 608-611.

(43) Saito, R.; Furukawa, M.; Dresselhaus, G.; Dresselhaus, M. S. Raman spectra of graphene ribbons. J. Phys.: Condens. Matter 2010, 22 (33), 334203.

(44) Talirz, L.; Söde, H.; Dumslaff, T.; Wang, S.; Sanchez-Valencia, J. R.; Liu, J.; Shinde, P.; Pignedoli, C. A.; Liang, L.; Meunier, V.; Plumb, N. C.; Shi, M.; Feng, X.; Narita, A.; Müllen, K.; Fasel, R.; Ruffieux, P. On-Surface Synthesis and Characterization of 9-Atom Wide Armchair Graphene Nanoribbons. ACS Nano 2017, 11 (2), $1380-1388$.

(45) Zhou, J.; Dong, J. Vibrational property and Raman spectrum of carbon nanoribbon. Appl. Phys. Lett. 2007, 91 (17), 173108.

(46) Li, X.; Cai, W.; An, J.; Kim, S.; Nah, J.; Yang, D.; Piner, R.; Velamakanni, A.; Jung, I.; Tutuc, E.; Banerjee, S. K.; Colombo, L.; Ruoff, R. S. Large-Area Synthesis of High-Quality and Uniform Graphene Films on Copper Foils. Science 2009, 324 (5932), 13121314.

(47) Liu, Z.; Wang, H. I.; Narita, A.; Chen, Q.; Mics, Z.; Turchinovich, D.; Kläui, M.; Bonn, M.; Müllen, K. Photoswitchable Micro-Supercapacitor Based on a Diarylethene-Graphene Composite Film. J. Am. Chem. Soc. 2017, 139 (28), 9443-9446.

(48) Chmiola, J.; Largeot, C.; Taberna, P.-L.; Simon, P.; Gogotsi, Y. Monolithic Carbide-Derived Carbon Films for Micro-Supercapacitors. Science 2010, 328 (5977), 480-483.

(49) Huang, P.; Lethien, C.; Pinaud, S.; Brousse, K.; Laloo, R.; Turq, V.; Respaud, M.; Demortière, A.; Daffos, B.; Taberna, P. L.; Chaudret, B.; Gogotsi, Y.; Simon, P. On-chip and freestanding elastic carbon films for micro-supercapacitors. Science 2016, 351 (6274), 691-695.

(50) Yuan, W.; Zhou, Y.; Li, Y.; Li, C.; Peng, H.; Zhang, J.; Liu, Z.; Dai, L.; Shi, G. The edge- and basal-plane-specific electrochemistry of a single-layer graphene sheet. Sci. Rep. 2013, 3, 2248.

(51) Tries, A.; Osella, S.; Zhang, P.; Xu, F.; Ramanan, C.; Kläui, M.; Mai, Y.; Beljonne, D.; Wang, H. I. Experimental Observation of Strong Exciton Effects in Graphene Nanoribbons. Nano Lett. 2020, 20 (5), 2993-3002.

(52) Ivanov, I.; Hu, Y.; Osella, S.; Beser, U.; Wang, H. I.; Beljonne, D.; Narita, A.; Müllen, K.; Turchinovich, D.; Bonn, M. Role of Edge Engineering in Photoconductivity of Graphene Nanoribbons. J. Am. Chem. Soc. 2017, 139 (23), 7982-7988. 
(53) Raza, H.; Kan, E. C. Armchair graphene nanoribbons: Electronic structure and electric-field modulation. Phys. Rev. B: Condens. Matter Mater. Phys. 2008, 77 (24), 245434.

(54) Richter, N.; Chen, Z.; Tries, A.; Prechtl, T.; Narita, A.; Müllen, K.; Asadi, K.; Bonn, M.; Kläui, M. Charge transport mechanism in networks of armchair graphene nanoribbons. Sci. Rep. 2020, 10 (1), 1988. 